K. Ishizaki

Nagoya Math. J.

Vol. 115 (1989), 199-207

\title{
ON SOME GENERALIZATIONS OF THEOREMS OF TODA AND WEISSENBORN TO DIFFERENTIAL POLYNOMIALS
}

\author{
KATSUYA ISHIZAKI \\ Dedicated to Professor Niro Yanagihara on his 60th birthday
}

\section{$\S 1$. Introduction}

We assume that the readers are familiar with the notations in Nevanlinna theory, see [2], [9].

Let $f$ be a nonconstant meromorphic function in the plane. We say that a function $h(r), 0 \leqq r \leqq \infty$, is $S(r, f)$ if

$$
h(r)=o(T(r, f))
$$

as $r \rightarrow \infty$, possibly outside a set of finite linear measure.

A meromorphic function $a(z)$ is said to be a small function for $f$ if

$$
T^{\prime}(r, a)=S(r, f) \text {. }
$$

Throughout this paper, we denote by $a, b_{0}, b_{1}, \cdots, a_{0}, a_{1}, \cdots$ small meromorphic functions for $f$.

Let

$$
\phi(z)=f^{n}+a_{n-1} f^{n-1}+, \cdots,+a_{1} f+a_{0} .
$$

E. Mues and N. Steinmetz [8] proved the following Theorem.

TheOREM A. Let $f$ be a meromorphic function. Assume that $\phi$ given by (1.1) satisfies

$$
\bar{N}(r, 0 ; \phi)=S(r, f) \quad \text { and } \quad \bar{N}(r, f)=S(r, f) .
$$

Then

$$
\phi=\left(f+a_{n-1} / n\right)^{n} .
$$

N. Toda [12] proved an extension of the Theorem A

THEOREM B. Let $f(z)$ be a meromorphic function and $\phi$ be given by

Received November 13, 1987. 
(1.1). If

$$
\lim _{r \rightarrow \infty} \sup _{r \in \bar{E} E}(\bar{N}(r, 0 ; \phi)+2 \bar{N}(r, f)) / T(r, f)<1 / 2,
$$

then we have

$$
\phi=\left(f+a_{n-1} / n\right)^{n} .
$$

Recently, Weissesnborn [14] proved the following theorem:

Theorem C. Let $f$ be a meromorphic function and $\phi$ be given by (1.1). Then we have that either

$$
\phi=\left(f+a_{n-1} / n\right)^{n}
$$

or

$$
T(r, f) \leqq \bar{N}(r, 0 ; \phi)+\bar{N}(r, f)+S(r, f) .
$$

In this note, we will extend these theorems to differential polynomials, instead of (mere) polynomial, of $f$.

We call, for a meromorphic function $f$,

$$
M[f]=a(z) f^{n_{0}}\left(f^{\prime}\right)^{n_{1}} \ldots\left(f^{(m)}\right)^{n_{m}}
$$

as a differential monomial in $f$ of degree $\gamma_{M}=n_{0}+\cdots+n_{m}$ and of weight $\Gamma_{M}=n_{0}+2 n_{1}+\cdots+(m+1) n_{m}$. We call

$$
P[f]=\sum_{\lambda \in I} M_{\imath}=\sum_{\lambda \in I} a_{\lambda}(z) f^{n_{0}}\left(f^{\prime}\right)^{n_{1}} \cdots\left(f^{(m)}\right)^{n_{m}}
$$

as a differential polynomial in $f$, where $a_{\lambda}$ are meromorphic functions and $I$ is a finite set of multi-indices $\lambda=\left(n_{0}, n_{1}, \cdots, n_{m}\right)$ for which $a_{\lambda} \neq 0$ and $n_{0}, n_{1}, \cdots, n_{m}$ are nonnegative integers. We define the degree $\gamma_{P}$ and weight $\Gamma_{P}$ of $P$ by

$$
\gamma_{P}=\max _{\lambda \in I} \gamma_{M_{\lambda}} \text { and } \max _{\lambda \in I} \Gamma_{M_{\lambda}} .
$$

If $P$ is a differential polynomial, then $P^{\prime}$ denotes the differential polynomial which satisfies

$$
P^{\prime}[f(z)]=\frac{d}{d z} P[f(z)]
$$

for any meromorphic function $f$. Note that $\gamma_{P^{\prime}}=\gamma_{P}$.

Steinmetz [11] investigated the value distribution of some differential polynomials in $f$. His result is as follows: put

$$
\Psi=f^{n} P[f]+Q[f],
$$


where $P$ and $Q$ are differential polynomials in $f$. Then

THEOREM D. Let $f$ be meromorphic function and $\Psi$ be given in (1.5) and $\Gamma_{Q} \leqq n-2$. If

$$
\bar{N}(r, 0 ; \Psi)=S(r, f)
$$

then

$$
m(r, f)+m(r, 0 ; f)+N_{1}(r, f)+N_{1}(r, 0 ; f)=S(r, f) .
$$

If, in (1.1), we replace $f$ by $f-a_{n-1} / n$, then we can write $\phi$ in (1.1) in the form

$$
\begin{aligned}
& \phi=f^{n}+Q[f], \\
& Q[f]=b_{n-2} f^{n-2}+\cdots+b_{1} f+b_{0} .
\end{aligned}
$$

The form (1.6) for polynomial corresponds to the form (1.5) with $\Gamma_{Q} \leqq n-2$ for differential polynomial.

In consideration of this Theorem D due to Steinmetz, we will prove here the following Theorems:

THEOREM 1. Let $f$ be a meromorphic function and $\phi$ be given in (1.6) and $Q[f] \neq 0$. Then

$$
2 T(r, f) \leqq \bar{N}(r, f)+\bar{N}(r, 0 ; f)+\bar{N}(r, 0 ; \phi)+S(r, f) .
$$

If $Q[0] \neq 0$, then

$$
n T(r, f) \leqq \bar{N}(r, f)+\bar{N}(r, 0 ; f)+\bar{N}(r, 0 ; Q)+\bar{N}(r, 0 ; \phi)+S(r, f)
$$

THEOREM 2. Let $f$ be a meromorphic function and $\Psi$ be given in (1.5). We suppose $Q[f] \neq 0$ and $\Gamma_{Q} \leqq n-2$. Then we have

$$
2 T(r, f) \leqq \bar{N}(r, f)+\bar{N}(r, 0 ; f)+\left(\gamma_{P}+1\right) \bar{N}(r, 0 ; \Psi)+S(r, f) .
$$

If further $m(r, P)=S(r, f)$, then

$$
2 T(r, f) \leqq \bar{N}(r, f)+\bar{N}(r, 0 ; f)+\bar{N}(r, 0 ; \Psi)+S(r, f) .
$$

\section{§ 2. Preliminary lemmas}

LEMma 1 ([2] [8] [11] [14]). Let $Q$ and $Q^{*}$ be differential polynomials in $f$ having coefficients $a_{j}$ and $a_{k}^{*}$. Suppose that $m\left(r, a_{j}\right)=S(r, f)$ and $m\left(r, a_{k}^{*}\right)=S(r, f)$, but we don't require that $T\left(r, a_{j}\right)=S(r, f)$ and $T\left(r, a_{k}^{*}\right)$ $=S(r, f)$. If $\gamma_{Q} \leqq n$ and 


$$
f^{n} Q^{*}[f]=Q[f]
$$

then

$$
m\left(r, Q^{*}[f]\right)=S(r, f) .
$$

Remark. Clunie proved his lemma under the stronger hypothesis that $T\left(r, a_{j}\right)=S(r, f)$ and $T\left(r, a_{k}^{*}\right)=S(r, f)$. Mues and Steinmetz [8] remarked that Clunie's proof does also work under the weaker assumption stated above. In particular, there might be coefficients of the form $f^{\prime} / f$ or, more generally, $\Psi^{\prime} / \Psi$ where $\Psi$ is the differential polynomial given by (1.0).

Lemma 2. If $P[f]$ is a differential polynomial and $\gamma_{P}=h$ then

$$
m(r, P) \leqq h m(r, f)+S(r, f) .
$$

Proof. Write

$$
P[f]=P_{h}[f]+\cdots+P_{0}[f]
$$

where $P_{j}[f](j=0,1, \cdots, h)$ are homogeneous polynomials with respect to $f, f^{\prime}, \cdots, f^{(m)}$, with degree $j . \quad P_{j}[f]$ is the sum of a finite number of terms [see 1],

$$
a(z)\left(f^{\prime} \mid f\right)^{n_{1}} \cdots\left(f^{(m)} / f\right)^{n_{m}} \cdot f^{j},
$$

where $j=n_{1}+\cdots+n_{m}$. Thus we can write

$$
P[f]=R_{h}[f] f^{h}+\cdots+R_{0}[f],
$$

where $R_{j}[f]=P_{j}[f] / f^{j}$ and hence

$$
m\left(r, R_{j}[f]\right)=S(r, f), \quad j=0,1, \cdots, h .
$$

Therefore we have

$$
\begin{aligned}
m(r, P[f]) & \leqq h m(r, f)+\sum_{j=0}^{h} m\left(r, R_{j} ; f\right) \\
& \leqq h m(r, f)+S(r, f) .
\end{aligned}
$$

Remark. Yang [13] proved above lemma under the condition $N(r, f)$ $=S(r, f)$.

\section{§ 3. Proof of Theorems 1 and 2}

Proof of Theorem 1. Write

$$
\phi=f^{n}+f^{m} Q_{1}[f]
$$


where

$$
0 \leqq m \leqq n-2, \quad Q_{1}[0] \neq 0, \quad \gamma_{Q_{1}}=\gamma_{Q}-m \leqq n-m-2 .
$$

Put $\psi=f^{n-m} / Q_{1}$ and apply the second fundamenntal Theorem to $\psi$. Then we obtain

$$
T(r, \psi) \leqq \bar{N}(r, \psi)+\bar{N}(r, 0 ; \psi)+\bar{N}(r,-1, \psi)+S(r ; \psi) .
$$

Since $\psi$ is a rational of $f$ with degree $n-m$, we apply the Mokhon'ko's theorem [6].

$$
T(r, \psi)=(n-m) T(r, f)+S(r, f) .
$$

Thus

$$
S(r, \psi)=S(r, f) \text {. }
$$

Each term on the right side of (3.1) are estimated as follows:

$$
\begin{aligned}
& \bar{N}(r, \psi) \leqq \bar{N}\left(r, 0 ; Q_{1}\right)+\bar{N}(r, f)+S(r, f), \\
& \bar{N}(r, 0 ; \psi) \leqq(r, 0 ; f)+S(r, f), \\
& \bar{N}(r,-1 ; \psi) \leqq \bar{N}(r, 0 ; \phi)+S(r, f), \\
& \bar{N}\left(r, 0 ; Q_{1}\right) \leqq(n-m-2) T(r, f)+S(r, f) .
\end{aligned}
$$

From (3.1)-(3.6)

$$
\begin{gathered}
(n-m) T(r, f) \leqq \\
+\bar{N}\left(r, 0 ; Q_{1}\right)+\bar{N}(r, f)+\bar{N}(r, 0 ; f) \\
+\bar{N}(r, 0 ; \phi)+S(r, f) .
\end{gathered}
$$

From (3.7) and (3.8), we obtain (1.7). If $Q[0] \neq 0$, that is $m=0, Q_{1}=Q$, then we get (1.8) by (3.8).

For the proof of Theorem 2, we follow some ideas given in [8], [11], [14].

Proof of Theorem 2. We may suppose $\psi \neq 0$, see [11]. Differentiating (1.5), we obtain

$$
f^{n-1} A=B
$$

with

$$
\begin{aligned}
& A=\left(\Psi^{\prime} \mid \Psi\right) f P-n f^{\prime} P+f P^{\prime} \\
& B=Q^{\prime}-\left(\Psi^{\prime} \mid \Psi\right) Q .
\end{aligned}
$$


By the Remark after the Lemma 1 , we look at $A$ and $B$ as differential polynomials in $f$ with coefficients having small proximity function and $\gamma_{B} \leqq n-2$.

We may suppose $A \neq 0$ [see 11]. By applying Lemma 1 we have

$$
\begin{aligned}
& m(r, A)=S(r, f), \\
& m(r, A f)=S(r, f),
\end{aligned}
$$

hence

$$
m(r, f) \leqq m(r, A f)-m(r, 0 ; A) \leqq m(r, 0 ; A)+S(r, f) .
$$

We define $\omega\left(z_{0}, f\right)$ as follows; if $z_{0}$ is a pole of $\nu$-th order for $f(z)$, then $\omega\left(z_{0}, f\right)=\nu$, and if $z_{0}$ is a regular point for $f(z)$, then $\omega\left(z_{0}, f\right)=0$. Let $z_{0}$ be a pole of $f$ and neither pole nor zero of coefficients of $P$ and Q. Put $\omega\left(z_{0}, f\right)=p$ and $\omega\left(z_{0}, Q\right)=k, 0 \leqq k \leqq p \Gamma_{Q} \leqq p(n-2)$. Write

$$
Q(z)=R /\left(z-z_{0}\right)^{k}+\cdots, \quad R \neq 0
$$

hence for $k \geqq 1$

$$
Q^{\prime}(z)=-k R /\left(z-z_{0}\right)^{k+1}+\cdots
$$

We have

$$
\Psi^{\prime}(z) / \Psi(z)=-n^{*} /\left(z-z_{0}\right)+\cdots, \quad\left(n^{*} \geqq n \geqq k+2\right) .
$$

From (3.11), (3.14), (3.15) and (3.16)

$$
B(z)=\left(n^{*}-k\right) R /\left(z-z_{0}\right)^{k+1}+\cdots
$$

For $k=0$, we have

$$
\omega\left(z_{0}, B\right)=1
$$

Thus

$$
\omega\left(z_{0}, B\right) \leqq k+1, \quad k \geqq 0 .
$$

If we have the development around $z_{0}$

$$
A(z)=S\left(z-z_{0}\right)^{\mu}+\cdots, \quad \mu \in Z, \quad S \neq 0,
$$

then from (3.9) and (3.17)

$$
p(n-1)-\mu \leqq k+1 \leqq p(n-2)+1 .
$$

hence 


$$
p-1 \leqq \mu
$$

Thus

$$
\omega\left(z_{0}, f\right)-1 \leqq \omega\left(z_{0}, 1 / A\right)
$$

By (3.10) and (3.18), if $z_{0}$ is a pole of $A$ and neither pole nor zero of coefficients of $P$ and $Q$ then, $z$ may not be pole of $f$. Thus $z_{0}$ is a zero of $\Psi$. And we see from (3.10) $\omega\left(z_{0}, A\right)$ is at most one. Therefore,

$$
\begin{aligned}
& \bar{N}(r, A) \leqq \bar{N}(r, 0 ; \Psi)+S(r, f), \\
& N_{1}(r, A)=S(r, f) .
\end{aligned}
$$

From (3.10)

$$
A=f P G
$$

with

$$
G=\left(\Psi^{\prime} \mid \Psi\right)-n\left(f^{\prime} \mid f\right)+\left(P^{\prime} \mid P\right) .
$$

Let $z_{1}$ be a zero of $f$ and neither pole nor zero of coefficients of $P$ and $Q$ then $\omega\left(z_{1}, G\right)$ is at most one by (3.23). Thus

$$
\omega\left(z_{1}, 1 / f\right)-1 \leqq \omega\left(z_{1}, 1 / A\right) .
$$

From (3.19) and (3.24)

$$
N_{1}(r, f)+N_{1}(r, 0 ; f) \leqq N(r, 0 ; A)+S(r, f) .
$$

From (3.22)

$$
m(r, A / f) \leqq m(r, P)+m(r, G) \leqq m(r, P)+S(r, f) .
$$

By the first fundamental theorem

$$
\begin{aligned}
m(r, f+(1 / f)) & =T\left(r,\left(f^{2}+1\right) / f\right)-N(r, f+(1 / f)) \\
& =2 T(r, f)-N(r, f)-N(r, 0 ; f)+O(1) \\
& =m(r, f)+m(r, 0 ; f)+O(1),
\end{aligned}
$$

hence

$$
\begin{aligned}
m(r, f)+ & m(r, 0 ; f)=m(r, f+(1 / f))+O(1) \\
& \leqq m\{r, A(f+(1 / f))\}+m(r, 0 ; A)+O(1) \\
& \leqq m(r, A f)+m(r, A / f)+m(r, 0 ; A)+O(1) .
\end{aligned}
$$

From (3.13), (3.26), (3.27) and Lemma 2 


$$
\begin{aligned}
m(r, f)+m(r, 0, f) & \leqq m(r, P)+m(r, 0 ; A)+S(r, f) \\
& \leqq h m(r, f)+m(r, 0 ; A)+S(r, f)
\end{aligned}
$$

from (3.14), we get

$$
m(r, f)+m(r, 0 ; f) \leqq(h+1) m(r, 0 ; A)+S(r, f) .
$$

By the first fundamental Theorem, (3.28) (3.25), (3.20) and (3.21), we obtain

$$
\begin{aligned}
2 T(r, f) & =m(r, f)+m(r, 0 ; f)+N_{1}(r, f)+N_{1}(r, 0 ; f) \\
& +\bar{N}(r, f)+\bar{N}(r, 0 ; f)+O(1) \leqq(h+1) m(r, 0 ; A)+N(r, 0 ; A) \\
& +\bar{N}(r, f)+\bar{N}(r, 0 ; f)+S(r, f) \leqq(h+1) T(r, A)+\bar{N}(r, f) \\
& +\bar{N}(r, 0 ; f)+S(r, f) \leqq(h+1) \bar{N}(r, A)+(h+1)\left\{N_{1}(r, A)\right. \\
& +m(r, A)\}+\bar{N}(r, f)+\bar{N}(r, 0 ; f)+S(r, f) \\
\leqq & (h+1) \bar{N}(r, 0 ; \Psi)+\bar{N}(r, f)+\bar{N}(r, 0 ; f)+S(r, f) .
\end{aligned}
$$

From this proof, if $m(r, P)=S(r, f)$, then we may put $h=0$ in (1.9). Thus Theorem 2 is proved.

\section{REFERENCES}

[1] H. S. Gopalakrishna and Subhas S. Bhoosnurmath, On the distribution of values of differential polynomials, Indian J. Pure appl. Math., 17 (3) (1986), 367-372.

[ 2 ] W. K. Hayman, Meromorphic functions, Oxford University Press 1964.

[ 3 ] K. Ishizaki, Deficient functions of meromorphic functions and powers, Technical Report of Mathematical Sciences, Chiba University, 1986, No. 21.

[4] K. Ishizaki, Exceptional values and deficient small functions of meromorphic functions and derivertives, Technical Report of Mathematical Sciences, Chiba University, 1987, No. 4.

[5] K. Ishizaki, On the value distribution of polynomials of Meromorphic functions, Technical Report of Mathematical Sciences, Chiba University, 1987, No. 5.

[6] A. Z. Mokhon'ko, On the Nevanlinna characteristics of some meromorphic functions in "Theory of functions, functional analysis and their applications", Izd-vo Khar'kovsk. Un-ta, No. 14 (1971), 83-87.

[ 7 ] A. Z. Mokhon'ko and V. D. Mokhon'ko, Estimates for the Nevanlinna characteristics of some classes of meromorphic functions and their applications to differential equations, Sib. Math. J., 15 (1974), 921-934.

[ 8 ] E. Mues and N. Steinmetz, The Theorem of Tumura-Clunie for meromorphic functions, J. London Math. Soc. (2), 23 (1981), 113-122.

[9] R. Nevanlinna, Analytic Functions, Springer-Verlag, New York, Heidelberg, Berlin, 1970.

[10] L. R. Sons, Deficiencies of monomials, Math. Z., 111 (1969), 53-68.

[11] N. Steinmets, Über die Nullstellen von Differential-polynomen, Math. Z., 176 (1981), 255-264.

[12] N. Toda, On an extension of the theorem of Tumura-Clunie, Comtemp. Math., 25 (1983), 215-219. 
[13] C. C. Yang, On deficiencies of differential polynomials, Math. Z., 116 (1970), 197-204.

[14] G. Weissenborn, On the theorem of Tumura-Clunie, Bull. London Math. Soc., 18 317-373 (1986).

Department of Mathematics

Tokyo National College of Technology

1220-2 Kunugida-cho, Hachioji,

Tokyo 193. Japan 